\title{
A Acessibilidade da Atenção Básica no Brasil na avaliação dos usuários
}

\section{Primary Health Care accessibility in Brazil: User "s evaluation}

\author{
Daniela Cristina Moreira Marculino de Figueiredo ${ }^{1,2}$ (D), Helena Eri Shimizu ${ }^{1}$, \\ Walter Massa Ramalho ${ }^{3}$ (D) \\ 'Departamento de Saúde Coletiva, Faculdade de Ciências da Saúde, Programa de pós graduação em saúde coletiva, \\ Universidade de Brasília (UnB) - Brasília (DF), Brasil. \\ 2 Departamento de Estatística, Programa de pós graduação em modelos de decisão e saúde, Universidade Federal da Paraíba- \\ João Pessoa (PB), Brasil. \\ ${ }^{3}$ Núcleo de Medicina Tropical, Faculdade de Ceilândia, Universidade de Brasilia (UnB) - Brasília (DF), Brasil.
}

Como citar: Figueiredo DCMM, Shimizu HE, Ramalho WM. A Acessibilidade da Atenção Básica no Brasil na avaliação dos usuários. Cad Saúde Colet, 2020;28(2):288-301. https://doi.org/10.1590/1414-462X202000020288

\section{Resumo}

Introdução: Embora sejam percebidos avanços quanto à ampliação na cobertura da Atenção Básica no Brasil, o acesso ou a acessibilidade do usuário na utilização desses serviços ainda é considerado como desafio. Objetivo: Descrever as dimensões da acessibilidade na atenção básica na avaliação dos usuários que participaram da avaliação externa do Programa Nacional de Melhoria do Acesso e da Qualidade na Atenção Básica, em 2012, nas macrorregiões do país. Método: Trata-se de um estudo descritivo, transversal. Foram utilizados os dados do módulo III, que trata da entrevista com os usuários na Unidade Básica de Saúde (UBS), especificamente do componente referente à utilização dos serviços de saúde. Participaram 65.391 usuários de 3.944 municípios. Resultados: Verificou-se que tanto a acessibilidade geográfica como a organizacional estão mais comprometidas nas regiões Norte e Nordeste. Quanto às barreiras de acessibilidade organizacional, constaram-se a forma de agendamento das consultas, a falta de possibilidade de escolha dos profissionais e os horários de atendimento restritos. Ademais, observou-se que boa parte dos usuários não consegue resolver as urgências na Unidade Básica de Saúde. Conclusão: Há avanços no alcance da acessibilidade, todavia nas regiões Norte e Nordeste é preciso maiores investimentos para melhorar, sobretudo, a acessibilidade organizacional.

Palavras-chave: atenção primaria à saúde; acesso aos serviços de saúde; avaliação em saúde; qualidade, acesso e avaliação da assistência à saúde; saúde pública.

\begin{abstract}
Background: Although advances are perceived in terms of expanding the coverage of Primary Care in Brazil, patient access or accessibility when using these services is still considered a challenge. Objective: This work aimed to describe some dimensions of accessibility in primary Health Care in Brazil of the users' evaluation who participate of the National Program for improving access and quality in primary health care, in 2012 in all the regions of the country. Method: It is a descriptive, cross-sectional study. It was used the data of the III module which collect with interview with users, data specifically of the component for the use of health services. 65.391 users of 3.944 municipalities participated of this study. Results: It was found problems with the geographical and organizational accessibility in the north and northeast regions. The organizational barriers were more evident in the scheduling consultations, in the lack of choice of professionals and the restricted opening hours. In addition, it was observed that most users cannot solve the emergency in the basic health unity. Conclusion: There was progress in the accessibility, on the other hand in the North and Northeast regions needs greater investments to improve the organizational accessibility.
\end{abstract}

Keywords: primary health care; health services accessibility; health evaluation; health care quality, access, and evaluation; public health.

Trabalho realizado no Programa de pós graduação em saúde coletiva, Universidade de Brasília (UnB), Brasília (DF).

Correspondência: Daniela Cristina Moreira Marculino de Figueiredo. E-mail: dla.moreira@yahoo.com.br

Fonte de financiamento: nenhuma.

Conflito de interesses: nada a declarar.

Recebido em: Ago. 16, 2017. Aceito em: Jun. 19, 2019

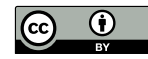

Este é um artigo publicado em acesso aberto (Open Access) sob a licença Creative Commons Attribution, que permite uso, distribuição e reprodução em qualquer meio, sem restrições desde que o trabalho original seja corretamente citado. 


\section{INTRODUÇÃO}

Alguns países da América do Sul têm investindo fortemente em processos de renovação da Atenção Primária à Saúde (APS), visando oferecer atenção integral à população, sobretudo mais equânime ${ }^{1}$. No Brasil, diversos esforços foram realizados, especialmente a revisão da Política Nacional de Atenção Básica, que colocou como aspectos centrais a melhoria do acesso e da qualidade.

O acesso ou a acessibilidade do usuário na utilização dos serviços ainda figura como um dos grandes desafios da Atenção Básica (AB). Donabedian² definiu o termo acessibilidade como a capacidade de produzir ofertas e de responder às necessidades de saúde de uma determinada população, observando-se as características dos serviços e dos recursos de saúde que auxiliam ou são obstáculos para a utilização pelos indivíduos. Starfield ${ }^{3}$ define a acessibilidade como elemento estrutural para o primeiro contato. Ela possibilita que as pessoas cheguem até os serviços de saúde e está relacionada com a disponibilidade e a capacidade de atenção ao primeiro contato. Geralmente a acessibilidade é definida como um componente da oferta ou como vínculo entre sujeitos e serviços de saúde ${ }^{4}$. Tudo aquilo que se interpõe entre a população e os serviços é considerado como "barreira" à acessibilidade, podendo ser caracterizada como barreira geográfica, econômica, organizacional e cultural/simbólica/psico/sociocultural4 .

No Brasil, estudos revelam aumento no acesso e na utilização dos serviços de saúde, especialmente pela população urbana (mesmo que com percentual ainda inferior ao observado em outros países) $)^{5}$. Além disso, há reconhecimento de que a consolidação da Estratégia Saúde da Família (ESF) tem sido associada aos efeitos benéficos sobre a equidade de acesso à saúde e maior vínculo entre usuários e equipes de saúde, bem como de ser a principal referência de cuidados ${ }^{5,6}$.

Nos últimos anos, diversos estudos de abrangência local e nacional têm abordado a análise do acesso e acessibilidade em saúde considerando os vários serviços e, especialmente, a $\mathrm{APS}^{7-10}$. Estudos que avaliam as dimensões da acessibilidade em saúde, nas diversidades regionais de um país continental como o Brasil são desejáveis, pois poderão melhor direcionar a elaboração de políticas públicas que contribuam para superar as dificuldades de utilização de serviços pela população.

Nessa perspectiva, o Ministério da Saúde instituiu o Programa Nacional de Melhoria do Acesso e da Qualidade da Atenção Básica (PMAQ-AB), que tem como objetivo induzir a instituição de processos que ampliem a capacidade das gestões federal, estaduais e municipais, bem como das equipes de $A B$, em ofertar serviços que assegurem maior acesso e qualidade e consonantes com as necessidades concretas da população.

O PMAQ-AB contou com quatro fases, sendo uma delas a avaliação externa, responsável pela coleta de informações relacionadas à infraestrutura das unidades, insumos e materiais, processo de trabalho e organização, articulação do cuidado junto à rede de atenção à saúde, além de informações relacionadas às experiências e opiniões dos usuários quanto à acessibilidade, utilização e satisfação dos serviços da $A B$.

É importante destacar a importância da escuta dos usuários sobre as dimensões da acessibilidade dos serviços de $A B$, pois são eles que vivem o drama diário da busca pela atenção à saúde. Assim, para este estudo, temos como questão: como os usuários que participaram da avaliação externa do PMAQ $A B$ avaliam os aspectos relacionados às dimensões da acessibilidade?

Este estudo se justifica, pois o primeiro ciclo do PMAQ traz informações inéditas e relevantes acerca da acessibilidade dos usuários aos serviços de $A B$, em âmbito nacional, portanto com capacidade para demonstrar as diferenças regionais, dentro do Brasil, que poderão subsidiar a reformulação das políticas públicas nessa área.

O presente estudo tem como objetivo descrever as dimensões da acessibilidade na Atenção Básica na avaliação dos usuários que participaram do Programa Nacional de Melhoria do Acesso e da Qualidade da Atenção Básica (PMAQ-AB) no ano de 2012, observando-se as disparidades nas macrorregiões do país.

\section{MÉTODO}

Trata-se de um estudo transversal, de caráter descritivo, que utilizou dados secundários, de domínio público, do PMAQ-AB, mais especificamente da avaliação externa do primeiro ciclo, realizado em 2012, por meio de consulta dos microdados disponíveis no portal do Departamento de Atenção Básica do Ministério da Saúde ${ }^{11}$. 
Foram utilizados os dados do módulo III, que trata da entrevista com os usuários na Unidade Básica de Saúde (UBS) com relação à sua experiência no acesso, utilização dos serviços de saúde e satisfação, sendo delimitado como recorte para este estudo o componente referente à utilização dos serviços de saúde pelos usuários ${ }^{11}$.

Os critérios de elegibilidade dos sujeitos para o estudo foram: usuários da $A B$ com pelo menos um atendimento na unidade nos últimos doze meses, maiores de 18 anos, que não tivessem passado pela consulta ou atendimento antes da entrevista, e vinculados à equipe de $A B$ (eAB). Embora a ESF seja o modelo prioritário de expansão e reorganização da $A B$, reconheceu-se que em algumas regiões do país existiam modalidades diferentes de $A B$ implantadas, onde a ESF não teve caráter substitutivo. Visando incorporar ao $P M A Q-A B$ essas equipes de $A B$, as quais foram denominadas de equipes de $A B$ parametrizadas, foram criados parâmetros, os quais consideraram núcleos mínimos de profissionais que compõem as equipes, carga horária de trabalho e organização que assegurassem os princípios e a Política Nacional de $A B$, a exemplo da territorialização e adscrição de pessoas por equipe de $A B^{12,13}$.

Participaram do estudo 65.391 usuários vinculados às $\mathrm{e} A B$, com adesão voluntária ao programa naquele ano, que correspondeu a 3.944 municípios (70\%) e 17.202 eAB (53,9\%).

A distribuição regional dos usuários participantes foi: 3.728 na Região Norte, 21.556 na Região Nordeste, 4.337 na Região Centro-Oeste, 25.406 na Região Sudeste e 10.364 na Região Sul.

Cada município poderia incluir todas ou apenas parte das suas equipes (eSF ou eAB parametrizadas). Por questões do cronograma de evolução logística, administrativa e orçamentária, foi fixado um teto máximo de adesão de 17.664 equipes de AB/SF no Brasil.

As principais variáveis estudadas foram: acessibilidade organizacional/funcional: horário de funcionamento; dias de funcionamento; outros horários de funcionamento da unidade; presença do médico na UBS ou em atividade no bairro durante todos os horários de funcionamento do serviço; possibilidade de escolha da equipe por parte do usuário; formas de marcação de consultas; e atendimento de urgência nas UBS. Acessibilidade geográfica: percepção da distância entre a unidade de saúde e a residência.

As descrições dos resultados basearam-se em comparação proporcionais das respostas das variáveis dos questionários, adotando-se como recorte as cinco regiões brasileiras. Os dados foram armazenados e organizados em planilha eletrônica Microsoft Office Excel 2010 para Windows ${ }^{\oplus}$. Para os cálculos dos intervalos de confiança das proporções utilizou-se uma significância de 0,05.

O estudo foi realizado a partir de dados secundários disponibilizados no sítio eletrônico do Departamento de Informática do Sistema Único de Saúde - Datasus, de domínio público, os quais preservam a identidade dos indivíduos. Assim, foram respeitados os princípios da ética em pesquisa envolvendo seres humanos, em conformidade com a Resolução n 466 , de 12 de dezembro de 2012, do Conselho Nacional de Saúde (CNS).

\section{RESULTADOS}

NaTabela 1 estão apresentadas as distribuições relativas às características sociodemográficas (gênero, idade, raça/cor) e econômicas (renda) dos usuários, segundo as regiões do Brasil.

Observou-se predominância do sexo feminino $(77,7 \%)$, sendo a idade média dos usuários de 49,3 anos. A raça/cor mais referida entre o total de entrevistados é a parda/mestiça (45,2\%), sobretudo na amostra da Região Norte. Contudo, na Região Sul, a raça/cor mais prevalente foi a branca $(74,7 \%)$ e com renda mensal familiar média de 3,94 salários mínimo, que se mostrou superior às demais regiões do país e da média nacional (1,87 salário mínimo).

Quanto ao recebimento do incentivo financeiro do Programa Bolsa Família (PBF), a maioria dos entrevistados $(61,1 \%)$ não era cadastrada no programa. Do percentual de participantes do estudo que referiram receber o benefício do PBF $(33,2 \%)$, destaca-se a Região Nordeste $(55,2 \%)$ e Norte $(48,1 \%)$.

Na Tabela 2 verifica-se que, para 67,1\% dos usuários, a Unidade Básica de Saúde (UBS) localiza-se próxima à residência, com destaque positivo para a Região Sudeste, com 69,7\%, e negativo para a Região Norte (59,6\%). Para a maior parte dos entrevistados a UBS funciona nos cinco dias da semana $(95,8 \%)$. Quanto à possibilidade de horários diferenciados, todos os usuários entrevistados identificaram o horário da tarde até às 18 horas e o período da noite, e alguns destacaram atendimentos aos sábados como importantes para facilitar o atendimento. 


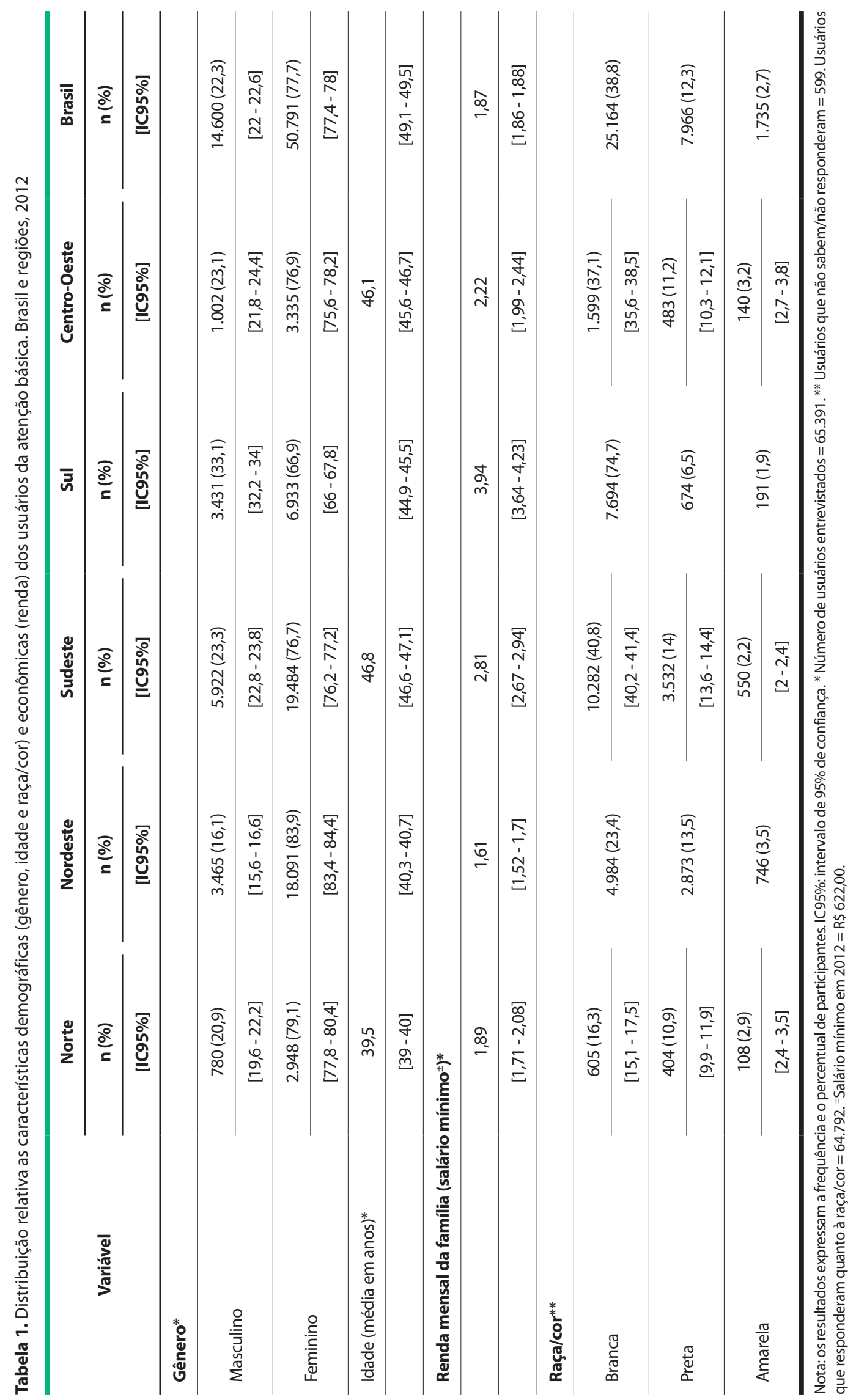




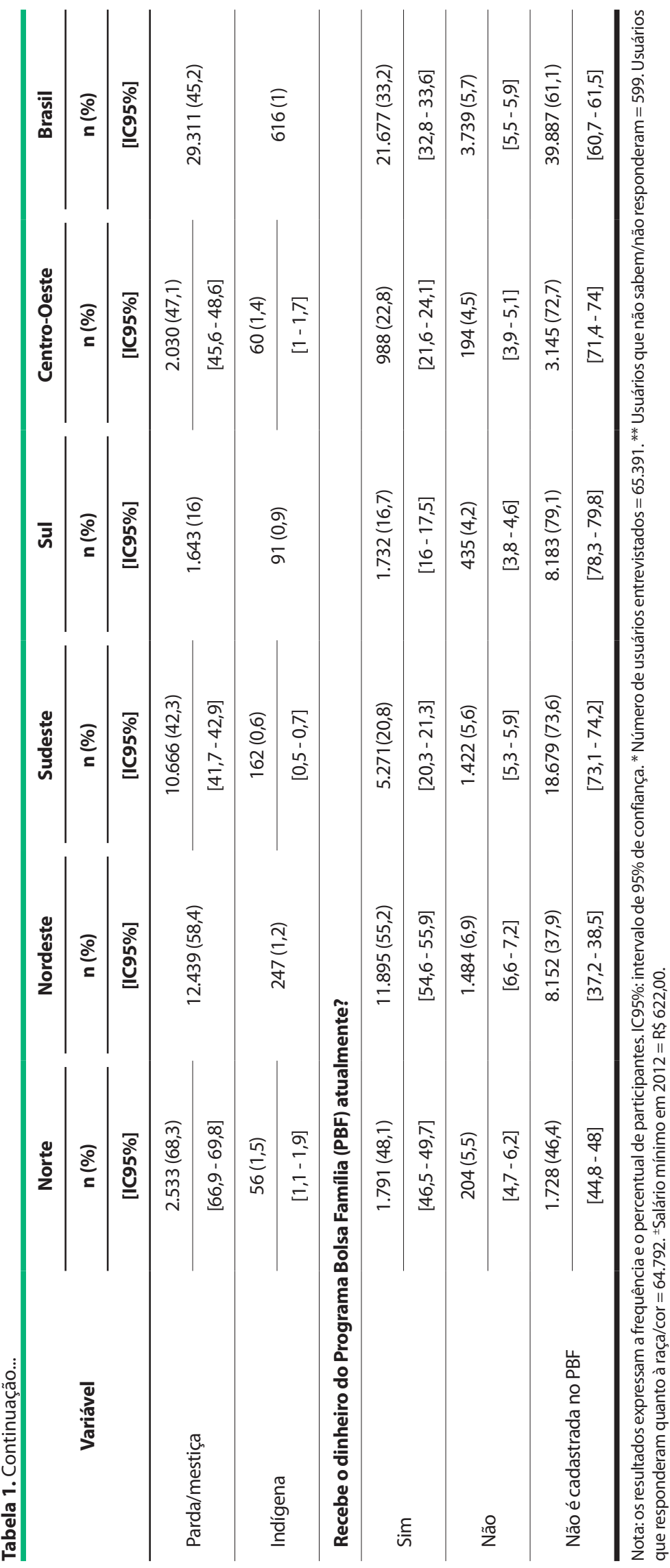




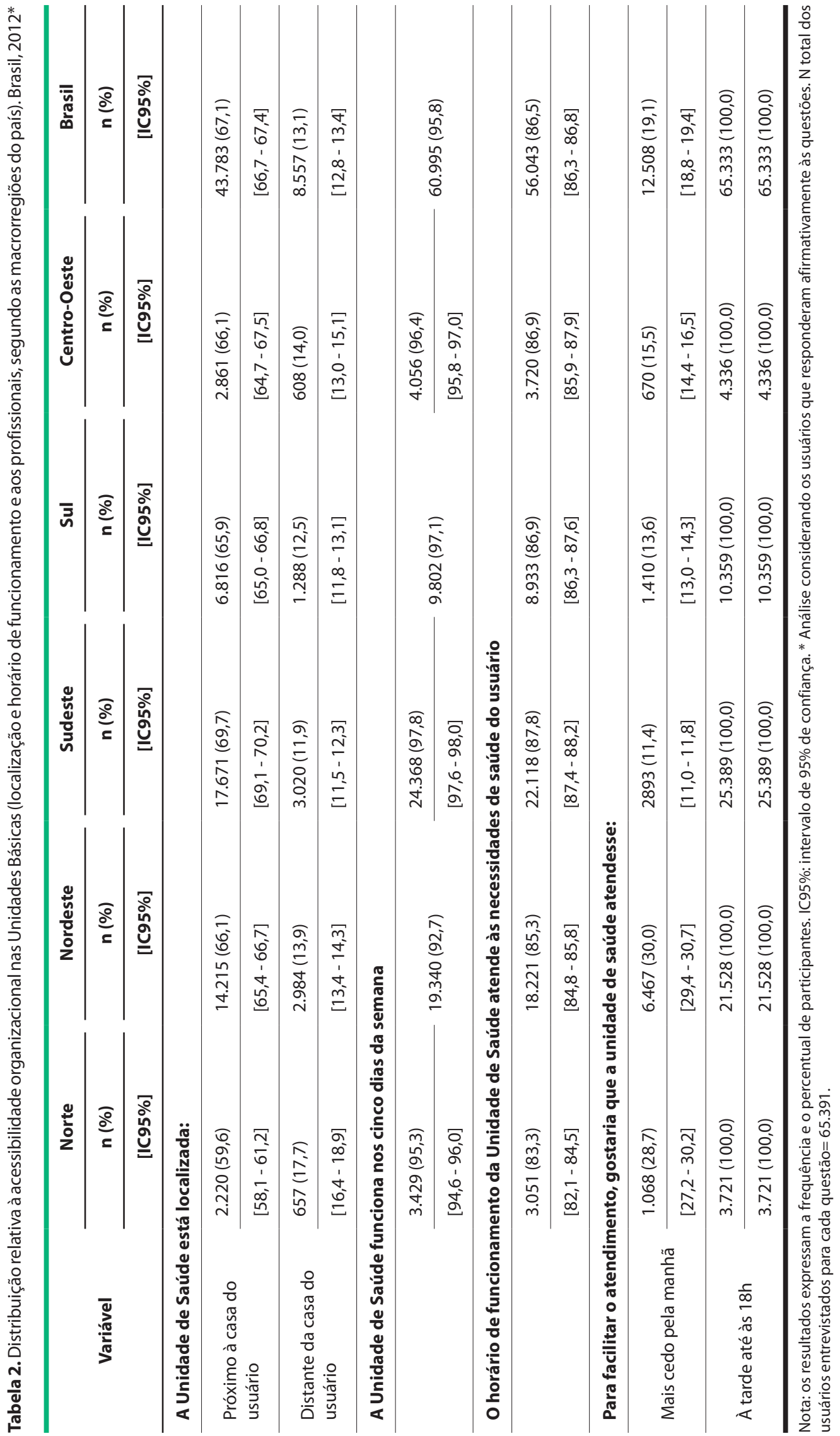




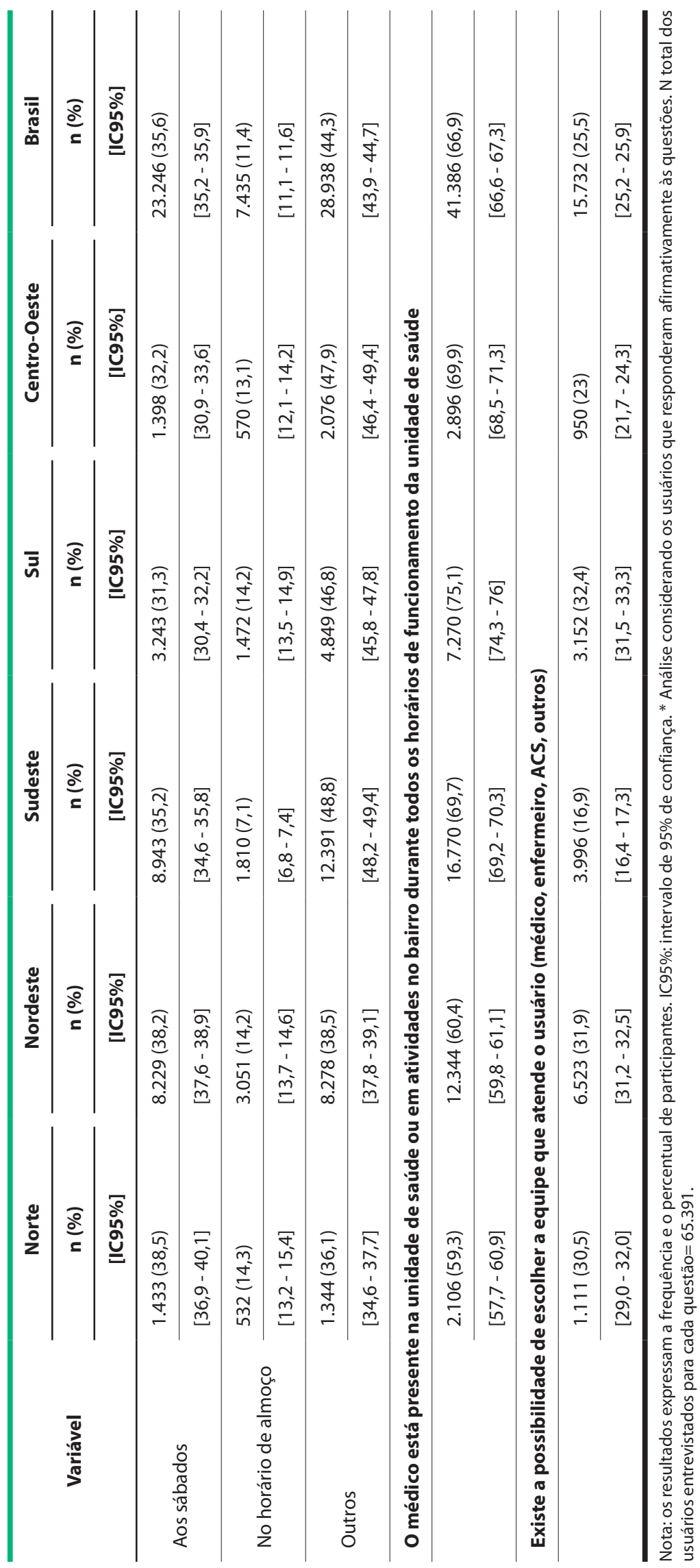


Ainda na Tabela 2 verificou-se que $66,9 \%$ dos usuários mencionaram a presença do profissional médico na UBS ou em atividades no bairro durante todos os horários de funcionamento, principalmente na Região Sul $(75,1 \%)$. Em comparação à Região Norte, este percentual foi de $59,3 \%$. Quanto à possibilidade de o usuário escolher a equipe de saúde que o atende (médico, enfermeiro, ACS ou outros), nas regiões Sul $(32,4 \%)$ e Nordeste $(31,9 \%)$ observaram-se maior frequência de respostas positivas, sendo a menor na região Sudeste (16,9\%).

Tabela 3. Distribuição relativa à acessibilidade organizacional quanto à marcação de consultas na Unidade Básica de saúde, segundo as macrorregiões do país. Brasil, 2012*

\begin{tabular}{|c|c|c|c|c|c|c|}
\hline \multirow{3}{*}{ Variável } & Norte & Nordeste & Sudeste & Sul & $\begin{array}{l}\text { Centro- } \\
\text { Oeste }\end{array}$ & Brasil \\
\hline & n (\%) & n (\%) & n (\%) & n (\%) & n (\%) & n (\%) \\
\hline & [IC95\%] & [IC95\%] & [IC95\%] & [IC95\%] & [IC95\%] & [IC95\%] \\
\hline \multicolumn{7}{|c|}{ Como ocorre a marcação da consulta na sua Unidade de Saúde? } \\
\hline \multirow{2}{*}{$\begin{array}{l}\text { Consulta } \\
\text { marcada pelo } \\
\text { telefone. }\end{array}$} & $24(0,6)$ & $90(0,4)$ & $497(2,0)$ & $696(6,7)$ & $157(3,6)$ & $1.464(2,2)$ \\
\hline & {$[0,4-0,9]$} & {$[0,3-0,5]$} & {$[1,8-2,1]$} & {$[6,2-7,2]$} & {$[3,1-4,2]$} & {$[2,1-2,4]$} \\
\hline \multirow{2}{*}{$\begin{array}{l}\text { Usuário vai } \\
\text { à unidade } \\
\text { e marca o } \\
\text { atendimento a } \\
\text { qualquer hora. }\end{array}$} & $732(19,6)$ & $3.566(16,6)$ & $9.716(38,3)$ & $2.670(25,8)$ & $1.641(37,8)$ & $1.8325(28)$ \\
\hline & {$[18,4-20,9]$} & {$[16,1-17,0]$} & {$[37,7-38,9]$} & {$[24,9-26,6]$} & {$[36,4-39,3]$} & {$[27,7-28,4]$} \\
\hline \multirow{2}{*}{$\begin{array}{l}\text { Usuário vai à } \\
\text { unidade, mas } \\
\text { tem que pegar } \\
\text { ficha. }\end{array}$} & $1.041(27,9)$ & $6.294(29,2)$ & $5.099(20,1)$ & $2.162(20,9)$ & $718(16,6)$ & $\begin{array}{c}15.314 \\
(23,4)\end{array}$ \\
\hline & {$[26,5-29,4]$} & {$[28,6-29,8]$} & {$[19,6-20,6]$} & {$[20,1-21,7]$} & {$[15,5-17,7]$} & {$[23,1-23,8]$} \\
\hline \multirow{2}{*}{$\begin{array}{l}\text { Usuário vai à } \\
\text { unidade e para } \\
\text { pegar ficha } \\
\text { é necessário } \\
\text { fazer fila antes } \\
\text { da unidade de } \\
\text { saúde abrir. }\end{array}$} & $1.076(28,9)$ & $8.178(38,0)$ & $5.206(20,5)$ & $4.241(40,9)$ & $1.283(29,6)$ & $\begin{array}{c}19.984 \\
(30,6)\end{array}$ \\
\hline & {$[27,4-30,3]$} & {$[37,3-38,6]$} & {$[20,0-21,0]$} & {$[40-41,9]$} & {$[28,2-30,9]$} & {$[30,2-30,9]$} \\
\hline \multirow{2}{*}{$\begin{array}{l}\text { O ACS marca a } \\
\text { consulta. }\end{array}$} & $689(18,5)$ & $2.633(12,2)$ & $3.917(15,4)$ & $216(2,1)$ & $147(3,4)$ & $7.602(11,6)$ \\
\hline & {$[17,2-19,7]$} & {$[11,8-12,7]$} & {$[15,0-15,9]$} & {$[1,8-2,4]$} & {$[2,9-3,9]$} & {$[11,4-11,9]$} \\
\hline \multirow{2}{*}{$\begin{array}{l}\text { Não faz } \\
\text { marcação de } \\
\text { consulta na } \\
\text { unidade de } \\
\text { saúde. }\end{array}$} & $56(1,5)$ & $209(1,0)$ & $195(0,8)$ & $94(1,0)$ & $45(1,1)$ & $599(0,9)$ \\
\hline & {$[1,1-1,9]$} & {$[0,8-1,1]$} & {$[0,7-0,9]$} & {$[0,8-1,2]$} & {$[0,8-1,4]$} & {$[0,9-1,0]$} \\
\hline \multirow{2}{*}{ Outro } & $109(2,9)$ & $575(2,7)$ & $756(3,0)$ & $280(2,7)$ & $345(8,0)$ & $2.065(3,2)$ \\
\hline & {$[2,4-3,5]$} & {$[2,5-2,9]$} & {$[2,8-3,2]$} & {$[2,4-3,0]$} & {$[7,2-8,8]$} & {$[3,0-3,3]$} \\
\hline
\end{tabular}

* número de usuários entrevistados = 65.353 (99,94\% da população estudada). IC95\%: intervalo de 95\% de confiança.

Na Tabela 3 pode ser verificado que, para marcar consultas na unidade de saúde, 30,6\% dos usuários referiram precisar ir à unidade, sendo necessário fazer fila antes da unidade de saúde abrir. Destaca-se a Região Sul (40,9\%), onde os usuários afirmaram ter que seguir esse procedimento para acessar o serviço de $A B$. Quanto à possibilidade de atendimento $a$ qualquer hora, foram mais prevalentes nos usuários residentes nas regiões Sudeste $(38,3 \%)$ e Centro-Oeste (37,8\%). Pode ser observado que ACS auxilia com maior frequência na marcação de consultas nas regiões Norte, $(18,5 \%)$, Sudeste $(15,4 \%)$ e Nordeste $(12,2 \%)$. 
Na Tabela 4 é possível observar que a população busca os serviços de emergência $(63,3 \%)$ ao apresentar um problema de saúde. Desses entrevistados, 65,3\% procuraram a UBS de referência para o seu atendimento. Aos que referiram não ter procurado a UBS em situações consideradas como urgências, os principais motivos apresentados foram: a UBS encontrar-se fechada no momento da urgência $(35,4 \%)$ ou ainda por este serviço não atender urgências $(31,8 \%)$.

Tabela 4. Distribuição relativa à acessibilidade organizacional quanto a atendimento de urgência na $A B$, segundo as macrorregiões do país. Brasil, 2012

\begin{tabular}{|c|c|c|c|c|c|c|}
\hline \multirow{3}{*}{ Variável } & Norte & Nordeste & Sudeste & Sul & $\begin{array}{l}\text { Centro- } \\
\text { Oeste }\end{array}$ & Brasil \\
\hline & n (\%) & n (\%) & n (\%) & n (\%) & n (\%) & n (\%) \\
\hline & [IC95\%] & [IC95\%] & [IC95\%] & [IC95\%] & [IC95\%] & [IC95\%] \\
\hline \multicolumn{7}{|c|}{ O usuário procurou atendimento quando apresentou problema de saúde que considerou uma urgência*: } \\
\hline \multirow{2}{*}{ Sim } & $2.411(65,0)$ & $\begin{array}{c}12.652 \\
(58,9)\end{array}$ & $\begin{array}{c}16.145 \\
(63,8)\end{array}$ & $7.365(71,3)$ & $2.645(61,2)$ & $\begin{array}{c}41.218 \\
(63,3)\end{array}$ \\
\hline & {$[63,5-66,5]$} & {$[58,2-59,5]$} & {$[63,2-64,4]$} & {$[70,4-72,2]$} & {$[59,8-62,7]$} & {$[62,9-63,6]$} \\
\hline \multirow{2}{*}{ Não } & $460(12,4)$ & $1.756(8,2)$ & $2.236(8,8)$ & $547(5,3)$ & $497(11,5)$ & $5.496(8,4)$ \\
\hline & {$[11,3-13,5]$} & {$[7,8-8,5]$} & {$[8,5-9,2]$} & {$[4,9-5,7]$} & {$[10,6-12,5]$} & {$[8,2-8,6]$} \\
\hline \multirow{2}{*}{$\begin{array}{l}\text { Não teve } \\
\text { problema de } \\
\text { urgência }\end{array}$} & $839(22,6)$ & $7.081(33,0)$ & $6.930(27,4)$ & $2.420(23,4)$ & $1.177(27,3)$ & $\begin{array}{c}18.447 \\
(28,3)\end{array}$ \\
\hline & {$[21,3-24,0]$} & {$[32,3-33,6]$} & {$[26,8-27,9]$} & {$[22,6-24,2]$} & {$[25,9-28,6]$} & {$[28,0-28,7]$} \\
\hline \multicolumn{7}{|c|}{ Procurou atendimento nesta unidade de saúde quando apresentou problema que considerou urgência ** } \\
\hline \multirow[t]{2}{*}{ Sim } & $1.407(58,4)$ & $8.024(63,4)$ & $\begin{array}{c}10.864 \\
(67,3)\end{array}$ & $5.157(70,0)$ & $1.463(55,3)$ & $\begin{array}{c}26.915 \\
(65,3)\end{array}$ \\
\hline & {$[56,4-60,3]$} & {$[62,6-64,3]$} & {$[66,6-68,0]$} & {$[69,0-71,1]$} & {$[53,4-57,2]$} & {$[64,8-65,8]$} \\
\hline \multirow[t]{2}{*}{ Não } & $1.004(41,6)$ & $4.628(36,6)$ & $5.281(32,7)$ & $2.208(30,0)$ & $1.182(44,7)$ & $\begin{array}{c}14.303 \\
(34,7)\end{array}$ \\
\hline & {$[39,7-43,6]$} & {$[35,7-37,4]$} & {$[32,0-33,4]$} & {$[28,9-31,0]$} & {$[42,8-46,6]$} & {$[34,2-35,2]$} \\
\hline \multicolumn{7}{|c|}{ Por que não procurou esta unidade de saúde para este atendimento?****} \\
\hline \multirow{2}{*}{$\begin{array}{l}\text { Porque precisa } \\
\text { chegar cedo }\end{array}$} & $13(1,3)$ & $50(1,1)$ & $49(0,9)$ & $33(1,5)$ & $16(1,4)$ & $161(1,1)$ \\
\hline & {$[0,6-2,0]$} & {$[0,8-1,4]$} & {$[0,7-1,2]$} & {$[1,0-2,0]$} & {$[0,7-2,0]$} & {$[1,0-1,3]$} \\
\hline \multirow{2}{*}{$\begin{array}{l}\text { Porque precisa } \\
\text { pegar ficha }\end{array}$} & $18(1,8)$ & $91(2,0)$ & $60(1,1)$ & $86(3,9)$ & $12(1,0)$ & $267(1,9)$ \\
\hline & {$[1,0-2,6]$} & {$[1,6-2,4]$} & {$[0,9-1,4]$} & {$[3,1-4,7]$} & {$[0,4-1,6]$} & {$[1,6-2,1]$} \\
\hline \multirow{2}{*}{$\begin{array}{l}\text { Porque não } \\
\text { atende sem } \\
\text { consulta } \\
\text { marcada }\end{array}$} & $49(4,9)$ & $164(3,5)$ & $294(5,6)$ & $109(4,9)$ & $42(3,6)$ & $658(4,6)$ \\
\hline & {$[3,5-6,2]$} & {$[3-4,1]$} & {$[5-6,2]$} & {$[4-5,8]$} & {$[2,5-4,6]$} & {$[4,3-4,9]$} \\
\hline \multirow{2}{*}{$\begin{array}{l}\text { Porque não tem } \\
\text { profissional na } \\
\text { unidade }\end{array}$} & $71(7,1)$ & $549(11,9)$ & $385(7,3)$ & $170(7,7)$ & $76(6,4)$ & $1.251(8,7)$ \\
\hline & {$[5,5-8,7]$} & {$[10,9-12,8]$} & {$[6,6-8,0]$} & {$[6,6-8,8]$} & {$[5,0-7,8]$} & {$[8,3-9,2]$} \\
\hline \multirow{2}{*}{$\begin{array}{l}\text { Porque não } \\
\text { atende urgência }\end{array}$} & $533(53,1)$ & $1.446(31,3)$ & $1.824(34,6)$ & $361(16,3)$ & $383(32,4)$ & $4.547(31,8)$ \\
\hline & {$[50,0-56,2]$} & {$[29,9-32,6]$} & {$[33,3-35,8]$} & {$[14,8-17,9]$} & {$[29,7-35,1]$} & {$[31,0-32,6]$} \\
\hline \multirow{2}{*}{$\begin{array}{l}\text { Porque a unidade } \\
\text { estava fechada } \\
\text { no momento da } \\
\text { urgência }\end{array}$} & $209(21,1)$ & $1.584(34,6)$ & $1.659(31,7)$ & $1.096(50,4)$ & $454(38,9)$ & $5.002(35,4)$ \\
\hline & {$[18,6-23,6]$} & {$[33,2-36,0]$} & {$[30,5-33,0]$} & {$[48,3-52,5]$} & {$[36,2-41,7]$} & {$[34,6-36,2]$} \\
\hline \multirow{2}{*}{ Outros } & $111(11,1)$ & $743(16,1)$ & $1.008(19,1)$ & $353(16,0)$ & $199(16,8)$ & $2.414(16,9)$ \\
\hline & {$[9,1-13,0]$} & {$[15,0-17,1]$} & {$[18,0-20,2]$} & {$[14,5-17,5]$} & {$[14,7-19,0]$} & {$[16,3-17,5]$} \\
\hline
\end{tabular}

* número de usuários entrevistados = 65.161 (99,64\% da população total estudada). IC95\%: intervalo de 95\% de confiança. ** número de usuários entrevistados (responderam "sim" à pergunta: procurou atendimento quando apresentou problema de saúde que considerou uma urgência): 41.218 ( $63 \%$ da população total estudada). *** número de usuários entrevistados (responderam "não" à pergunta: procurou atendimento nesta unidade de saúde quando apresentou problema que considerou urgência): 14.303 (34,7\% da população que procurou atendimento em algum serviço de saúde quando apresentou uma situação que considerou urgência). 


\section{DISCUSSÃO}

O estudo revelou que a população feminina utiliza mais os serviços da $A B$ do que os homens. Resultados semelhantes têm sido encontrados em outros estudos ${ }^{5,14}$. As mulheres buscam mais os serviços de saúde para exames de rotina ou para cuidado preventivo. Destarte, as ofertas disponíveis, sobretudo nas unidades básicas de saúde, estão, muitas vezes, relacionadas ao período gravídico e puerperal. Os homens buscam mais o cuidado curativo ${ }^{15} \mathrm{e}$ recorrem aos serviços de saúde quando não conseguem exercer o seu papel de trabalhador, pelo maior número de morbidades ${ }^{16}$ ou a partir de intercorrências graves.

Quanto à renda das famílias dos usuários da $A B$, a população da Região Nordeste apresentou a menor renda (1,61 salários mínimos). Observou-se ainda que os entrevistados em sua maioria não recebiam o benefício do Programa Bolsa Família (PBF), o que demonstra que não é somente a população mais pobre que utiliza os serviços de $A B$. As regiões Nordeste e Norte concentram o maior percentual de beneficiários do PBF.

Em relação à acessibilidade geográfica, verificou-se que para a maioria dos usuários, a unidade de saúde encontra-se próxima às suas residências, contudo a distância ainda figura como barreira de acesso aos cuidados de saúde nas diferentes regiões do país ${ }^{17}$. A grande dimensão territorial associada aos problemas de acesso ocasiona o isolamento geográfico, sobretudo nas áreas rurais. A Região Norte possui $30 \%$ da população vivendo em meio rural, que apresenta grande dificuldade para utilizar os serviços de saúde, muitas vezes concentrados nas capitais ou em municípios com maior porte populacional. A escassez ou mesmo ausência de UBS para a população desses territórios compromete a garantia da saúde como direito constitucional, que reflete em indicadores de morbimortalidade mais críticos, em comparação às demais regiões do país ${ }^{18-21}$. Segundo estudo ${ }^{6}$ realizado também com dados do PMAQ-AB, $38,4 \%$ dos usuários viviam há mais de $1 \mathrm{~km}$ de distância de uma UBS, sendo $42,5 \%$ de usuários mais pobres.

A maioria dos usuários afirmou que as unidades funcionam nos cinco dias da semana, o que facilita o acesso aos cuidados. Análise realizada por Fausto et al..$^{22}$ demonstra que o maior porte populacional estava diretamente proporcional à quantidade de UBS com funcionamento nos cinco dias da semana, com exceção dos municípios com população acima de 500 mil habitantes. Contudo, todos os entrevistados gostariam, para facilitar o atendimento, que a unidade de saúde abrisse à tarde, após as 18 h e à noite, e alguns afirmaram que desejariam que a unidade abrisse aos sábados. Estes achados assemelham-se ao encontrado em outros estudos, em que os usuários referiam a necessidade de ampliação dos horários para facilitar o acesso, sobretudo da população trabalhadora ${ }^{23}$.

Observou-se neste estudo dificuldade de acesso da população ao profissional médico durante todos os horários de funcionamento da unidade de saúde, mais acentuadamente nas regiões Norte e Nordeste, o que também foi identificado em outros estudos para dimensionar a força de trabalho do médico no Brasil24. O Programa Mais Médicos foi criado para suprir essas carências, notadamente em área remotas, com aumento de número de municípios com mais de um médico por 1000 habitantes, que passou de 163 para 348 no período de 2013 a $2015^{25}$. Ademais, esse programa melhorou o acesso aos médicos em todos os dias e horários, especialmente onde havia médicos cubanos que apresentam forte senso comunitário ${ }^{26}$.

Ainda quanto à acessibilidade organizacional, evidenciou-se que o fato das eSF serem fixas em um determinado território dificulta a escolha dos profissionais pelos usuários. Em experiências internacionais, que consideram as questões relativas à vinculação dos usuários com os serviços existentes no território, existe a possibilidade de os usuários escolherem a equipe de saúde ou os profissionais, mediante uso de outros critérios como a proximidade do trabalho ${ }^{27-31}$. Acredita-se que liberdade de escolha das equipes ou dos profissionais pelos usuários contribui para maior adesão ao tratamento e aos cuidados.

Verificou-se neste estudo que o tempo de espera para a marcação das consultas não consideradas de urgência tende a ser demorado, com superlotação para o atendimento médico e pouco agendamento para o profissional enfermeiro, como também demonstra estudo realizado em Florianópolis-SC, em 47 centros de saúde ${ }^{32}$. 
Quanto à forma de agendamento de consultas, a do tipo porta aberta, a qualquer hora que o usuário chegar à UBS foi relativamente baixo e mais prevalente nas regiões Sudeste, Centro-Oeste e Sul. Outra forma de acesso à consulta, a mais referida pelos usuários, foi através de ficha/senha, principalmente nas regiões Nordeste e Norte, onde é necessário o usuário formar fila antes da unidade de saúde abrir, sendo esse padrão de resposta mais observado nos usuários das regiões Sul e Nordeste do país. Outros estudos corroboram este achado ${ }^{33,34}$, que além de ser considerado importante obstáculo ao acesso, é também limitado do ponto de vista do cuidado integral, uma vez que considera apenas as queixas e percepções das desordens biomédicas, não valorizando as demais necessidades da população.

Com isso, poderá haver tendência à cultura da medicalização do sofrimento humano, que poderá gerar incessante busca da população por cuidados médicos, que por sua vez, não conseguem absorver todas as demandas, gerando insatisfação entre os usuários e profissionais de saúde 32 .

Nesse sentido, é fundamental que seja fomentado um novo modelo de atenção, considerando o trabalho interdisciplinar, a clínica ampliada e os saberes dos diversos profissionais e, sobretudo, o protagonismo dos usuários. Com isso, busca-se romper com o paradigma da atenção à saúde centrada em procedimentos e na medicalização dos problemas ${ }^{35}$, já que, na maioria das vezes, esse modelo é pouco resolutivo, hierárquico do ponto de vista relacional e com baixa responsabilização por parte dos usuários. Uma forma de organizar o acesso aos serviços da UBS, e principalmente às consultas, seria o acolhimento, através de uma escuta atenta aos problemas de saúde para dar respostas às necessidades dos usuários, estabelecer vínculos de cuidado e confiança baseados em abordagens centradas na pessoa e não na doença. Essa prática pode auxiliar na definição da $A B$ como o lócus de cuidado preferencial no sistema de saúde, superando a negativa da atenção pela ausência das fichas.

Por fim, o estudo revelou que aproximadamente $35 \%$ dos usuários entrevistados não buscaram a UBS em situações consideradas como urgência, revelando assim que ainda há dificuldades para a $A B$ incorporar essa responsabilidade. Em experiências internacionai ${ }^{36} \mathrm{com}$ APS forte busca-se a incorporação do atendimento nas UBS para que não haja nenhum tempo de espera, sendo os usuários atendidos no mesmo dia por algum profissional ou em até 48 horas pelo médico da unidade de saúde.

Outro estudo realizado também com dados do PMAQ-AB22 revelou que quanto menor o porte populacional do município, maiores são as possibilidades da UBS estar fechada, sendo este um obstáculo de acesso relacionado ao horário de funcionamento. No entanto, esses municípios atendem mais situações de urgências que os municípios de maior porte populacional, segundo os usuários entrevistados. A Política Nacional de Atenção às Urgências, por meio da constituição da Rede de Urgência e Emergência (RUE), reforça enquanto objetivo atribuído à $A B$ o primeiro cuidado às urgências e emergências, em ambiente adequado, até $a$ transferência ou encaminhamento a outros pontos de atenção, quando necessário.

Entretanto, além do problema do horário de funcionamento das unidades de saúde já citado anteriormente, que não permite o acesso dos usuários em situações de urgência ou de demanda não agendada, ainda é comum a percepção por parte dos profissionais e da população que este nível de atenção deve ser responsável apenas por desenvolver ações de promoção e proteção da saúde e prevenção dos agravos.

Há também a percepção por parte dos gestores e trabalhadores que o número elevado de pessoas sob a responsabilidade de uma equipe, em boa parte das regiões do Brasil, em comparação aos países que demonstram apresentar forte $\mathrm{APS}^{37}$, impossibilita a atenção integral, incluindo o acesso às urgências ${ }^{38}$. E ainda, que o maior acesso na APS está relacionado a menores taxas de internação por emergência hospitalar ${ }^{39}$. Como consequência, o que se observa na maioria dos serviços, ou em boa parte deles, é pouco acesso às urgências, levando-as a buscar atenção em serviços de pronto atendimento ou emergências hospitalares, gerando superlotação neles, com demandas que deveriam ser atendidas na $A B$, deslegitimando-a socialmente ${ }^{40}$.

Há que se destacar ainda que os países com boa acessibilidade possuem cobertura de APS acima de $90 \%$ e número de população por equipe de saúde inferior a 2.000 pessoas. No Brasil, 
entre 2012 e 2016, contava-se respectivamente com 54,8\% e 60,9\% de cobertura da eSF e um número superior de população adscrita por equipe de saúde.

Quanto às limitações do estudo, podemos citar o desenho, de corte transversal, e a amostragem de conveniência - quatro usuários por unidade, em municípios que formalizaram a adesão e contratualização ao programa. Todavia pela abrangência nacional e homogeneidade metodológica do estudo, os resultados possibilitam a percepção quanto aos obstáculos e avanços relacionados às dimensões da acessibilidade na $A B$ no Brasil bem como contribui para apontar algumas questões relacionadas às desigualdades regionais no acesso.

A acessibilidade em saúde na $A B$ ainda é grande desafio, tendo-se as barreiras geográficas como importantes obstáculos para boa parte da população. Desse modo, devem se continuar investindo na ampliação da cobertura.

O acesso dificultado pela organização do serviço evidencia a necessidade de romper com as práticas de cuidado centradas no acesso a partir das fichas e filas. Ademais, é preciso organizar formas de ofertas de saúde mais inclusivas, como o horário de atendimento mais flexível.

A urgência nas UBS é outro tema que requer profundas reflexões, em especial sobre o seu papel nas RAS, fundamental para melhor organização do sistema de serviços de saúde nas regiões de saúde.

Por fim, espera-se que sejam fomentados outros estudos a partir dos dados do PMAQ-AB, bem como o uso dos seus resultados, enquanto substrato para o planejamento das ações de saúde, pelas três instâncias de gestão do SUS, visando a qualidade e o fortalecimento da AB.

\section{REFERÊNCIAS}

1. Giovanella L, Almeida PF, Vega Romero R, Oliveira S, Tejerina Silva H. Panorama de la Atención Primaria de Salud en Suramérica: concepciones, componentes y desafíos. Saúde Debate. 2015;39(105):300-22. http://dx.doi.org/10.1590/0103-110420151050002002.

2. Donabedian A. Aspects of medical care administration. Cambridge: Harvard University Press; 1973.

3. Starfield B. Atenção Primária: equilíbrio entre necessidades de saúde, serviços e tecnologia. Brasília: UNESCO, Ministério da Saúde; 2002.

4. Landini F, González Cowes V, Damore E. Hacia un marco conceptual para repensar la accesibilidad cultural. Cad. Saúde Pública, 2014;30(2):231-44.

5. Dourado I, Medina MG, Aquino R. The effect of the Family Health Strategy on usual source of care in Brazil: data from the 2013 National Health Survey (PNS 2013). Int J Equity Health. 2016;15(1):1-10. http://dx.doi. org/10.1186/s12939-016-0440-7. PMid:27852265.

6. Rasella D, Machado DB, Castellanos ME, Paim J, Szwarcwald CL, Lima D, et al. Assessing the relevance of indicators in tracking social determinants and progress toward equitable population health in Brazil. Glob Health Action. 2016;9(1):29042. http://dx.doi.org/10.3402/gha.v9.29042. PMid:26853898.

7. Alves MGM, Casotti E, Oliveira LGD, Machado MTC, Almeida PF, Fonseca MP, et al. Fatores condicionantes para o acesso às equipes da Estratégia Saúde da Família no Brasil. Saúde Debate. 2014;38(N. esp.):34-51.

8. Oliveira APC, Gabriel M, Dal Poz MR, Dussault G. Desafios para assegurar a disponibilidade e acessibilidade à assistência médica no Sistema Único de Saúde. Cien Saude Colet. 2017;22(4):1165-80. http://dx.doi. org/10.1590/1413-81232017224.31382016. PMid:28444043.

9. Albuquerque MSV, Lyra TM, Farias SF, Mendes MFM, Martelli PJL. Acessibilidade aos serviços de saúde: uma análise a partir da Atenção Básica em Pernambuco. Saúde Debate. 2014;38(N. esp.):182-194.

10. Barbiani R, Junges JR, Rigon Dalla Nora C, Asquidamini F. A produção científica sobre acesso no âmbito do Sistema Único de Saúde do Brasil: avanços, limites e desafios. Saude Soc. 2014;23(3):855-68. http:// dx.doi.org/10.1590/S0104-12902014000300010.

11. Brasil. Ministério da Saúde. Departamento de Atenção Básica. Microdados da avaliação Externa PMAQAB: usuários Brasil [Internet]. Brasília: Ministério da Saúde; 2012 [citado em 2017 jan 23]. Disponível em: http://aps.saude.gov.br/ape/pmaq/ciclo1/

12. Brasil. Ministério da Saúde. Departamento de Atenção Básica. Programa Nacional de Melhoria do Acesso e da Qualidade da Atenção Básica (PMAQ): manual instrutivo [Internet]. Brasília: Ministério da Saúde; 2012 [citado em 2017 jan 23]. Disponível em: http://189.28.128.100/dab/docs/publicacoes/geral/ manual_instrutivo_pmaq_site.pdf 
13. Poças KC, Freitas LRS, Duarte EC, Poças KC, Freitas LRS, Duarte EC. Censo de estrutura da Atenção Primária à Saúde no Brasil (2012): estimativas de coberturas potenciais. Epidemiol Serv Saude. 2017;26(2):275-82. http://dx.doi.org/10.5123/S1679-49742017000200005. PMid:28492769.

14. Vieira KLD, Gomes VLO, Borba MR, Costa CFS. Atendimento da população masculina em unidade básica saúde da família: motivos para a (não) procura. Esc Anna Nery. 2013;17(1):120-127.

15. Moura EC, Santos W, Medeiros das Neves AC, Gomes R, Shwarz E. Atenção à saúde dos homens no âmbito da Estratégia Saúde da Família. Ciênc \& Saúde Coletiva. 2014;19(2):429-38. http://dx.doi.org/10.1590/141381232014192.05802013.

16. Malta DC, Bernal RTI, Lima MG, Araujo SSC, Silva MMA, Freitas MIF, Barros, MBA. Doenças crônicas não transmissíveis e a utilização de serviços de saúde: análise da Pesquisa Nacional de Saúde no Brasil. Ver. Saúde Publica. 2017;51(1 Suppl):1s-4s.

17. Protásio APL, Gomes LB, Machado LS, Valença AMG. Satisfação do usuário da Atenção Básica em Saúde por regiões do Brasil: $1^{\circ}$ ciclo de avaliação externa do PMAQ-AB. Ciênc. Saúde Colet; 2017;22(6):1829-44.

18. Viellas EF, Domingues RMSM, Dias MAB, Gama SGN, Theme-Filha MM, Costa JV, et al. Assistência pré-natal no Brasil. Cad Saude Publica. 2014;30(Suppl.):85-100. http://dx.doi.org/10.1590/0102-311X00126013.

19. Bühler HF, Ignotti E, Silva Neves SMA, Souza Halcon S. Análise espacial de indicadores integrados determinantes da mortalidade por diarreia aguda em crianças menores de 1 ano em regiões geográficas. Ciênc Saúde Coletiva. 2014;19(10):4131-40. http://dx.doi.org/10.1590/1413-812320141910.09282014.

20. Klafke A, Duncan BB, Rosa RDS, Moura LD, Malta DC, Shmidt MI. Mortalidade por complicações agudas do diabetes melito no Brasil, 2006-2010. Epidemiol Serv Saude. 2014;23(3):455-62. http://dx.doi.org/10.5123/ S1679-49742014000300008.

21. Girianelli VR, Gamarra CJ, Silva GAE. Contrastes na mortalidade por câncer feminino. Rev Saude Publica. 2014;48(3):459-67. http://dx.doi.org/10.1590/S0034-8910.2014048005214. PMid:25119941.

22. Fausto MCR, Giovanella L, Mendonça MHM, Seidl H, Gagno J. A posição da Estratégia Saúde da Família na rede de atenção à saúde na perspectiva das equipes e usuários participantes do PMAQ-AB. Saúde em debate. 2014;38(N. esp.):13-33.

23. Reis RS, Coimbra LC, Silva AAM, Santos AM, Alves MTSSB, Lamy ZC, et al. Acesso e utilização dos serviços na Estratégia Saúde da Família na perspectiva dos gestores, profissionais e usuários. Cien Saude Colet. 2013;18(11):3321-31. http://dx.doi.org/10.1590/S1413-81232013001100022. PMid:24196897.

24. World Health Organization. Increasing access to health workers in remote and rural areas through improved retention: global policy recommendations. Genebra:WHO; 2010.

25. Santos LMP, Oliveira A, Trindade JS, Barreto ICHC, Palmeira PA, Comes Y, et al. Implementation of the Mais Médicos Program to achieve universal primary healthcare coverage among vulnerable populations in Brazil. Bull World Health Organ. 2017;95:103-12. http://dx.doi.org/10.2471/BLT.16.178236. PMid:28250510.

26. Giovanella $L$, Stegmüller K. Tendências de reformas da atenção primária à saúde em países europeus. J Manag Prim Health Care. 2014;5(1):101-13. http://dx.doi.org/10.14295/jmphc.v5i1.203.

27. Marchildon GP. Canada: Health system review. Health Syst Transit. 2013;15(1):1-179. PMid:23628429.

28. Barros P, Machado S, Simões J. Portugal: Health system review. Health Syst Transit. 2011;13(4):1-156. PMid:22222781.

29. García-Armesto S, Abadía-Taira MB, Durán A, Hernández-Quevedo C, Bernal-Delgado E. Spain: Health system review. Health Syst Transit. 2010;12(4):1-295. PMid:21224176.

30. Ferré F, de Belvis AG, Valerio L, Longhi S, Lazzari A, Fattore G, et al. Italy: Health system review. Health Syst Transit. 2014;16(4):1-168. PMid:25471543.

31. Ringard Å, Sagan A, Sperre Saunes I, Lindahl AK. Norway: Health system review. Health Syst Transit. 2013;15(8):1-162. PMid:24434287.

32. Tesser CD, Norman AH. Repensando o acesso ao cuidado na Estratégia Saúde da Família. Saúde Soc. São Paulo. 2014;23(3):869-83.

33. Sarti TD. A marcação de consulta médica em uma unidade de saúde da família: uma etnografia a respeito dos conflitos entre usuários e profissionais de saúde. Rev. APS. 2014;17(3):362-72.

34. Sousa FOS, Medeiros KRD, Gurgel J, Dantas G, Albuquerque PCD. Do normativo à realidade do Sistema Único de Saúde: revelando barreiras de acesso na rede de cuidados assistenciais. Cien Saude Colet. 2014;19(4):1283-93. http://dx.doi.org/10.1590/1413-81232014194.01702013. PMid:24820611.

35. Coelho MO, Jorge MSB. Tecnologia das relações como dispositivo do atendimento humanizado na atenção básica à saúde na perspectiva do acesso, do acolhimento e do vínculo. Cien Saude Colet. 2009;14(1 Suppl):1523-31. http://dx.doi.org/10.1590/S1413-81232009000800026. PMid:19750362. 
36. Salisbury C, Banks J, Goodall S, Baxter H, Montgomery A, Pope C et al. Na evaluation of Advanced Acess in general practice: final report. Bristol: NHS; 2007.

37. Giovanella L, Escorel S, Lobato LDVC, Noronha JCD, Carvalho AID. Políticas e sistema de saúde no Brasil. Rio de Janeiro: Fiocruz; 2008.

38. Soares SS, Lima LD, Castro ALB. O papel da atenção básica no atendimento às urgências: um olhar sobre as políticas. J ManagPrim Health Care. 2014;5(2):170-7. http://dx.doi.org/10.14295/jmphc.v5i2.213.

39. Cowling TE, Harris M, Watt $H$, Soljak M, Richards E, Gunning E, et al. Access to primary care and the route of emergency admission to hospital: retrospective analysis of national hospital administrative data. BMJ Qual Saf. 2015;25(6):432-40. http://dx.doi.org/10.1136/bmjqs-2015-004338. PMid:26306608.

40. Oliveira LH, Mattos RAD, Souza AISD. Cidadãos peregrinos: os "usuários" do SUS e os significados de sua demanda a prontos-socorros e hospitais no contexto de um processo de reorientação do modelo assistencial. Cien Saude Colet. 2009;14(5):1929-38. http://dx.doi.org/10.1590/S1413-81232009000500035. PMid:19851606. 